\title{
Photo Catalytic Silver Modified Orthodontic Brackets - An Innovative Method for Prevention of White Spot Lesions
}

\author{
Rizwan Gilani¹, Pavan Bajaj², Nikhil Mankar³, Rozina Vishnani", Pallavi Daigavane ${ }^{5}$, Priyanka Niranjane ${ }^{6}$ \\ ${ }^{1}$ Department of Orthodontics, Sharad Pawar Dental College (SPDC), Datta Meghe Institute of Medical Sciences \\ (DMIMS(Du)), Sawangi (M), Wardha, Maharashtra, India. ${ }^{2}$ Department of Periodontics, Sharad Pawar Dental \\ College (SPDC), Datta Meghe Institute of Medical Sciences (DMIMS(Du)), Sawangi (M), Wardha, Maharashtra, \\ India. ${ }^{3}$ Department of Endodontics, Sharad Pawar Dental College (SPDC), Datta Meghe Institute of Medical \\ Sciences (DMIMS(Du)), Sawangi (M), Wardha, Maharashtra, India. ${ }^{4}$ Department of Oral and Maxillofacial \\ surgery, Bharti Vidyapeeth (DU), Sangli, Maharashtra, India. ${ }^{5}$ Department of Orthodontics, Sharad Pawar \\ Dental College (SPDC), Datta Meghe Institute of Medical Sciences (DMIMS(Du)), Sawangi(M), Wardha, \\ Maharashtra, India. ${ }^{6}$ Department of Orthodontics, Sharad Pawar Dental College(SPDC), Datta Meghe Institute of \\ Medical Sciences (DMIMS(Du)), Sawangi (M), Wardha, Maharashtra, India.
}

\section{ABSTRACT}

\section{BACKGROUND}

Association between fixed orthodontic therapy and enamel decalcification causing periodontal disease and enamel decalcification is a known problem of orthodontic treatment. The root cause of this is the fact that brackets provide an ideal environment for bacteria to accumulate and multiply. Hence modifying the surface of brackets with some photocatalytic antibacterial substance could help in prevention of this side-effect. The current study was thus planned to evaluate the anti-adherence of bacteria to photocatalytic silver coated brackets for the prevention of white spot lesions.

\section{METHODS}

After obtaining ethical clearance from the institutional ethics committee, 40 metal brackets of upper central incisor were taken. These brackets were divided into group 1 \& group 2 each containing twenty brackets. Group 1 (control group) consisted of plain metal brackets while group 2 (experimental group) consisted of silver coated brackets. Both the groups were subjected to laboratory bacterial tests to assess the bacterial adhesion to brackets and then statistical analysis was done to obtain results.

\section{RESULTS}

Silver modified brackets showed around $25 \%$ less adhesion of bacteria as compared with plain brackets.

\section{CONCLUSIONS}

Modifying orthodontic brackets by coating them with photocatalytic silver could prove to be an innovative and effective method in prevention of white spot lesions after fixed orthodontic therapy.

\section{KEY WORDS}

Photo - Catalysis, Silver, Bacterial Adhesion, White Spots, Brackets
Corresponding Author: Dr. Rizwan Gilani, Associate Professor, Department of Orthodontics, Sharad Pawar Dental College (SPDC), (DMIMS (du), Sawangi (M), Wardha, Maharashtra, India.

E-mail: drrizwangilani@gmail.com

DOI: $10.14260 / j e m d s / 2020 / 607$

How to Cite This Article:

Gilani R, Bajaj P, Mankar N, et al. Photo catalytic silver modified orthodontic brackets- an innovative method for prevention of white spot lesions. J Evolution Med Dent Sci 2020;9(38):2787-2790, DOI: 10.14260/jemds/2020/607

Submission 08-05-2020,

Peer Review 14-08-2020,

Acceptance 20-08-2020,

Published 21-09-2020.

Copyright (C) 2020 Rizwan Gilani et al. This is an open access article distributed under Creative Commons Attribution License [Attribution 4.0 International (CC BY 4.0)] 


\section{BACKGROUND}

The specialty of orthodontics has evolved enormously along with drastic changes in biomechanics and material science from the day Angle first introduced fixed appliance. Recent advances in orthodontic material science \& technology have come a long way. In last few decades, there has been an enormous increase in demand for orthodontic treatment from patients of all age groups. Also the patient's and doctor's expectations from outcomes of orthodontic treatment is increasing day by day. Clinicians always wish to give the best orthodontic treatment possible with the most stable results. But, in spite of the various developments in orthodontic materials and techniques, the finishing of orthodontic treatment is hindered by the risk of development of periodontal diseases and white spot lesions on teeth around the appliances. This still remains a common side effect of orthodontic treatment especially in patients who have very poor oral hygiene. To the orthodontist, health of the surrounding para - dental tissue is of utmost importance as they contain the required substance that helps the orthodontist not only to move the teeth to new positions in the oral cavity but also perhaps alter the form of the alveolar bone.

Fixed orthodontic appliance therapy deals with the correction of malaligned teeth by the application of light continuous force on teeth by the arch wires through the orthodontic brackets. Orthodontic brackets along with arch wires and elastics remain in in the oral cavity for almost one and half years. Orthodontic brackets provides ideal condition for the growth of bacteria due to their complex design and irregular and sharp surface. ${ }^{1}$ Furthermore the difficulty in maintaining oral hygiene to orthodontic patients due to presence of brackets and wires increases the colonization of bacteria around the brackets even more. This bacterial colonization over the period of time results in plaque accumulation, enamel demineralization finally progressing to white spot lesions (wsl). ${ }^{2}$ patients undergoing braces treatment commonly becomes victims of this side - effect which is clearly visible after debonding of orthodontic brackets. ${ }^{3-5}$ Orthodontic brackets are proven to have very high critical surface tension and hence they have the tendency to accumulate bacteria around them. ${ }^{6}$ The irregular and complex design of orthodontic brackets further hinders proper cleaning around it thus increasing the bacterial accumulation. ${ }^{7}$ stainless steel brackets are the most commonly used and preferred brackets by the orthodontists for treating their patients however elevated plaque adhesion, increased bacterial activity and thus low $\mathrm{p}^{\mathrm{h}}$, around these stainless steel brackets leading to white spots on teeth has been well proven. 8,9

White spot lesions are matter of concern for orthodontist as well as orthodontic patients due to their unaesthetic and unhealthy appearance. Also, these white spot lesions are irreversible in nature and hence prevention of these unhealthy and unaesthetic lesions is considered as one of the most important objectives of successful orthodontic treatment. ${ }^{10}$ One of the methods to reduce bacterial accumulation around orthodontic brackets is by organic degradation process. Degradation of organic compounds by photo catalysis for decontamination using semiconductor particles has proved to be quite effective and among these particles, silver has been proved to be the best substance on account of its chemically stable properties. Various other substances like titanium dioxide, silver di-oxide, zinc oxide, tungsten oxide, etc. Are considered effective for this process, ${ }^{11}$ however silver has been considered to be ideal and most useful substance for this process. ${ }^{10,11}$ Hence, Applying the concept of photo - catalysis in orthodontics for prevention of bacterial accumulation could prove to be an innovative concept for prevention of white spot lesions, Therefore the above study was planned to evaluate the anti-adherence of bacteria to photocatalytic silver coated orthodontic brackets for the preventing the development of white spot lesions.

\section{METHODS}

The above study was an in-vitro study. In order to assess the anti - adherent properties of orthodontic brackets, 40 stainless steel pre - adjusted edgewise orthodontic brackets of $3 \mathrm{M}$ Unitek of upper right central incisor were selected after consulting a statistician. The sample size was selected after taking into consideration the minimum sample size required to obtain a significance value. These orthodontic brackets were subdivided into two groups, group 1 (control group) \& group 2 (experimental group). Each group containing twenty brackets. Group 1 - 20 brackets (control group) having plain (uncoated) metal orthodontic brackets while group $2-20$ brackets (experimental group) having silver coated orthodontic brackets. After incubating these brackets in culture broth. Both the groups were subjected to laboratory bacterial tests to assess the adhesion of bacteria to brackets by weight change comparison.

Surface modification (coating) of SS orthodontic brackets with silver was done with electromagnetic sputtering method using "Tecport coating unit" at Indian Institute of Science (IISC) Bengaluru. Sputtering process removes superficial particles from the target substance by sputtering it with positively charged ion from inert gas discharge and depositing it above the substrate as a micro film. Then the silver coated brackets were subjected to oxidation in an open-air furnace at $500^{\circ} \mathrm{C}$ in order to achieve a uniform coating of silver di - oxide.

For assessing the adhesion of bacteria to the orthodontic brackets, the orthodontic brackets were first cleaned in ultrasonic cleaner for removal of surface impurities and then autoclaved. The orthodontic brackets were then pre- weighed on digital balance and packed in air tight Eppendorf tubes.

Cultured broth inoculated to the concentration of $10 \%$ was used and $0.5-1 \mathrm{ml}$ of this solution was then put into each of the Eppendorf tubes. The orthodontic brackets were then submerged in it for incubation under UV light inside the laminar air flow chamber for 24 hrs. Bacteria adhered orthodontic brackets were then removed and kept in formaldehyde solution to immobilize the cells. After a thorough washing with distilled water, the orthodontic brackets were subjected to drying for $24 \mathrm{hrs}$. The weight change of the brackets was again recorded.

\section{Statistical Analysis}

Inferential \& descriptive statistics was done. Assessment was done at $5 \%$ significance. Student $\mathrm{t}$ - test was used to calculate the significance value of study. Leven $1 \mathrm{~s}$ test was used to 
evaluate the homogeneity of variance. The software used for statistical analysis was, Stata 10.1 was used for the data analysis, and Microsoft office was used for graphs $\&$ tables

\section{RESULTS}

\begin{tabular}{|c|c|c|c|c|c|c|}
\hline & & & Differenc & $95 \%$ & & \\
\hline & Betore & After & (\%) & CL & 1 & $P$ \\
\hline $\begin{array}{c}\text { Plain } \\
\text { Brackets }\end{array}$ & $0.0702 \pm 0.00$ & $\begin{array}{c}0.0743 \pm 0.0003 \\
(0.0738-0.0749)\end{array}$ & $\begin{array}{c}-0.004 \\
(5.85 \%)\end{array}$ & -0.00 & 61.125 & $<0.001^{* *}$ \\
\hline $\begin{array}{c}\text { Silver } \\
\text { Modified } \\
\text { Brackets }\end{array}$ & $\begin{array}{c}0.0782 \pm 0.00 \\
(0.0782- \\
0.0782)\end{array}$ & $\begin{array}{c}0.0799 \pm 0.0002 \\
(0.0795-0.0802)\end{array}$ & $\begin{array}{l}-0.002 \\
(2.21 \%)\end{array}$ & - 0.00 & 38.637 & $<0.001^{* *}$ \\
\hline Value of $t$ & $\mathrm{~T}=02.5+10$ & $\mathrm{~T}=70.55$ & - & - & - & - \\
\hline Value of $p$ & $<0.001^{* *}$ & $<0.001^{* *}$ & & & & \\
\hline & Table 2 & Weight Compa & son of $B$ & ckets & & \\
\hline
\end{tabular}

Orthodontic brackets coated with the photocatalytic silver showed comparatively less adherence effect of $S$. mutans compared with the uncoated orthodontic brackets. The bacteria's that sticked to silver coated orthodontic brackets were much less when compared to the uncoated orthodontic brackets. Apart from the reduction in bacterial adhesion to its surface, the silver coated orthodontic brackets also had a bactericidal effect on $S$. mutans which is known to cause dental caries and periodontal diseases. It can be seen from the above table that plain brackets showed more increase in the weight as compared to the silver coated brackets thus proving that bacterial adhesion is more in uncoated brackets.

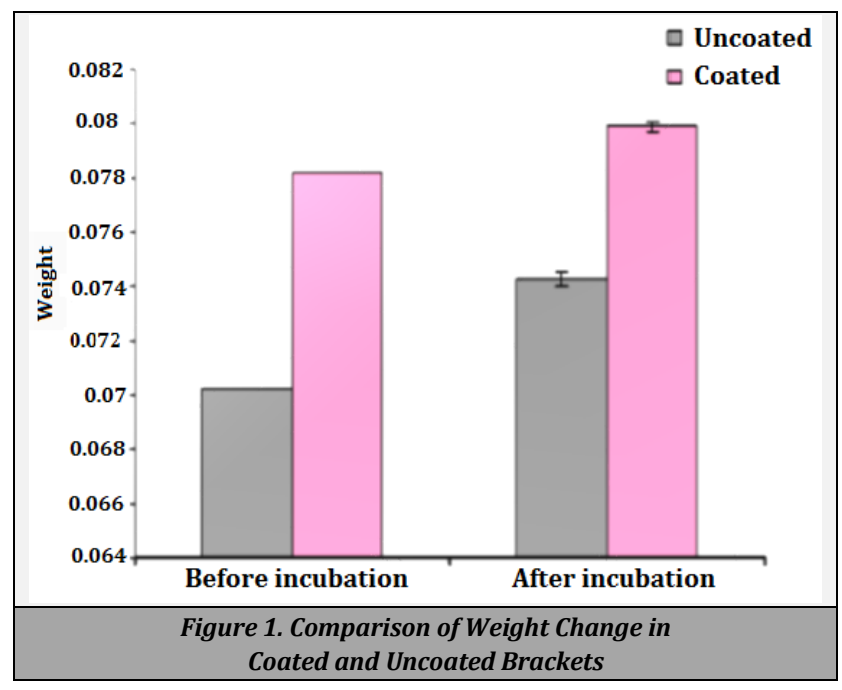

\section{DISCUSSION}

Several parameters are said to influence the physical, chemical and biological characteristics of dental plaque, thus favouring the development of dental caries or periodontal diseases. One of the environmental changes that leads to the formation of dental plaque and also alters the severity of it, is the placement of orthodontic brackets and bands. The inherent irregularities of brackets and bands provide additional surface for the collection of food and debris. ${ }^{1}$ Brackets can alter the ecological environment in the oral cavity by development of new retention areas available for bacterial colonization and retention of the debris. ${ }^{2}$ This increased supply of debris for the bacteria permits the fluent growth of bacteria. The complex design of fixed orthodontic appliance also protects the plaque from the action of brushing, mastication and salivary flow. ${ }^{1}$ Brackets are considered one of the important part of the orthodontic therapy but due to their complicated design and high surface tension they provide a good environment for the bacteria to accumulate and multiply. The prevalence of enamel demineralization on labial surface of teeth after fixed orthodontic treatment involves up to $60 \%$ cases. Prevalence of such white spot lesions around orthodontic brackets has been observed within 1 month of appliance placement.3,4.

Literature on the management of oral hygiene in orthodontic patients have been concentrated on the effects of different oral hygiene protocols ${ }^{12,13}$. Various Chemical solutions and antibiotics have been used as bactericidal agents against $S$. mutans bacteria to reduce plaque related diseases, including dental caries ${ }^{14}$. However, these chemical solutions and antibiotic agents sometimes can have adverse effects such as nausea, vomiting, diarrhoea, and tooth staining 15,16 . Though Chlorhexidine mouthwashes, as an adjunct to tooth brushing, is effective in the control of gingival inflammation, its prolonged use may cause problems such as staining of teeth. ${ }^{16}$ Bad taste, tooth staining, and temporary alteration of taste most of the time leads to low patient compliance. Hence altering the surface of orthodontic brackets with photocatalytic antibacterial silver can help in prevention of this side - effect. Antibacterial and anti - adherent properties of titanium coated brackets has already been proven. ${ }^{17}$ Hence it can be accepted that reducing adherence of bacteria to the surface of the brackets could be an innovative method for prevention of enamel decalcification \& wsl. ${ }^{12}$ Silver has been a known antibacterial drug and is also used for coating in industries due to its anti - corrosive properties by creating a passive layer.13 Hence the concept of coating with photocatalytic silver was thought. One of the Randomized Clinical Trial revealed that that silver alloy catheters are significantly more effective in preventing urinary tract infections due to its antibacterial property. ${ }^{14}$ In an in - vitro study conducted by Arun Mahaska et al in $2015^{15}$ it was proved that modifying the surface of orthodontic wires with photocatalytic silver has drastically reduced the accumulation of plaque on its surface. Hence the same concept was thought to be applied to brackets ${ }^{16}$. Apart from these, mouthwashes can cause some amount of corrosion of the wires and brackets that are placed in patient's mouth. This leads to loss of physical properties of the wires \& brackets and also release of the nickel ions which have been shown to be toxic and cause of allergic reactions in the human body.

Various studies have been done in which antimicrobial substances were mixed in the bonding system. These Materials showed antibacterial effect by sustained release of the added agent. But leaching of the antimicrobials from these material have various disadvantages, such as decreased mechanical properties of the carrier substance over a period of time, short lived effectiveness and possible toxicity to human health. Above a certain caries activity level, neither an increase in the frequency of tooth brushing nor in the dose of added fluoride is suitable to totally stop the demineralization process, especially in the high risk individuals. The concept of using silver for coating brackets instead of titanium comes from the 
limitations of previous studies. ${ }^{17}$ The first being that titanium is very expensive which will create hesitation among the practitioners from using these brackets, second when the brackets were coated with titanium, it was found to have lost their shininess and they appear slightly blackish which will definitely be an aesthetic concern. Thus, coating of brackets with silver was considered. In spite of the major developments in orthodontic materials brackets in this form are not available in the market. So coating silver on stainless steel orthodontic brackets which are readily available in the market is chosen. Also the unavoidable problem of demineralization and plaque accumulation around the brackets during orthodontic treatment has not been overcome. The prevention and reduction of periodontal diseases and white spot lesions during fixed orthodontic therapy still remains one of the greatest challenges for the practicing orthodontist.

\section{CONCLUSIONS}

Modifying Stainless Steel Orthodontic brackets by coating it with photocatalytic silver could prove to be an innovative method in prevention of enamel demineralization and white spot lesions in patients undergoing fixed orthodontic treatment due to its anti-bacterial and anti-adherent properties.

\section{Limitation}

It is a lab based study, clinical trials would be required to prove its efficacy and permit its use in day to day practice.

Financial or Other Competing Interests: None.

\section{REFERENCES}

[1] Balenseifen JW, Madonia JV. Study of dental plaque in orthodontic patients. J Dent Res 1970;49(2):320-4.

[2] Hägg U, Kaveewatcharanontet P, Samaranayak YH. Et al. The effect of fixed orthodontic appliances on the oral carriage of Candida species and Enterobacteriaceae. Euro J Orthod 2004;26(6):623-9.

[3] Gorelick L, Geiger AM, Gwinnett AJ. Incidence of white spot formation after bonding and banding. Am J Orthod 1982;81(2):93-8.

[4] Thote AM, Sharma K, Uddanwadiker RV, et al. Pure intrusion of a mandibular canine with segmented arch in lingual orthodontics: a numerical study with 3dimensional finite element analysis. Biocybernet Biomed Eng 2017;37(3):590-98.
[5] Thote AM, Sharma K, Uddanwadier RV, et al. Optimum pure intrusion of a mandibular canine with the segmented arch in lingual orthodontics. Biomed Mater Eng 2017;28(3):247-56.

[6] Artun J, Brobakkan BO. Prevalence of carious white spots after orthodontic treatment with multibonded appliances. Euro J Orthod 1986;8(4):229-34.

[7] Eliades T, Eliades G, Brantley WA. Microbial attachment on orthodontic appliances: I. Wettability and early pellicle formation on bracket materials. Am J Oethod Dentofacial Orthop 1995;108(4):351-60.

[8] Rosenbloom RG, Tinanoff N. Salivary Streptococcus mutans levels in patients before, during and after orthodontic treatment. Am J Orthod Dentofacial Orthop 1991;100(1):35-7.

[9] Ahn SJ, Lim BS, Lee SJ. Prevalence of cariogenic streptococci on incisor brackets detected by polymerase chain reaction. Am J Orthod Dentofacial Orthop 2007;131(6):736-41.

[10] Corbett JA, Brown LR, Keene HJ, et al. Comparison of Streptococcus mutans concentrations in non-banded and banded orthodontic patients. J Dent Res 1981;60(12):1936-42.

[11] Shah AG, Shetty PC, Ramachandra CS, et al. In vitro assessment of photocatalytic titanium oxide surface modified stainless steel orthodontic brackets for antiadherent and antibacterial properties against Lactobacillus acidophilus. Angle Orthod 2011;81(6):1028-35.

[12] Oh KT, Joo UH, Park GH, et al. Effect of silver addition on the properties of nickel-titanium alloys for dental application. J Biomed Mater Res B Appl Biomater 2006;76(2):306-14.

[13] Saint S, Elmore JG, Sullivan SD, et al. The efficacy of silver alloy-coated urinary catheters in preventing urinary tract infection: a meta-analysis. Am J Med 1998;105(3):236-41.

[14] Robertson MA, Kau CH, English JD, et al. MI paste plus to prevent demineralization in orthodontic patients: a prospective randomized controlled trial. Am J Orthod Dentofacial Orthop 2011;140(5):660-8.

[15] Mhaske AR, Shetty PC, Bhat NS, et al. Antiadherent and antibacterial properties of stainless steel and NiTi orthodontic wires coated with silver against lactobacillus acidophilus--an in vitro study. Prog Orthod 2015;16:40.

[16] Jung WK, Koo HC, Kim KW, et al. Antibacterial activity and mechanism of action of the silver ion in Staphylococcus aureus and Escherichia coli. Appl Environ Microbiol 2008;74(7):2171-8.

[17] Lakshmi S, Renganathan R, Fujita S, et al. Study on TiO2mediated photocatalytic degradation of methylene blue. J Photochem Photobiol A: Chemistr 1995;88(2-3):163-7. 